\title{
Variation of microwave dielectric properties in the glucose biosensor system
}

\begin{abstract}
Glucose biosensor is generally based on the reaction between glucose and glucose oxidase, which produces gluconic acid and hydrogen peroxide. The gluconic acid is a conductive material, while hydrogen peroxide has polar molecules. This article examines the changes of dielectric properties due to the conductive loss below $2 \mathrm{GHz}$ and dipole orientation of above that frequency of this reaction. The difference between the dielectric loss of glucose solution and the dielectric loss of glucose-enzyme reaction can be related to the glucose concentration in the samples, such as orange juice, black grape juice, sugarcane juice, and sapodilla juice. A good sensitivity to these differences due to the effect of ionic conductivity and dipole orientation was found at 1 and $16.44 \mathrm{GHz}$, respectively. The minimum detection limit of glucose concentration in the proposed technique was about $0.01 \mathrm{M}(0.20 \mathrm{~g} / 100 \mathrm{ml})$ with an optimum reaction ratio of about 1:1 between the enzyme solution and the glucose solution. This technique could benefit the future development of microwave biosensor by which both ionic conductivity and dipole effects can be considered simultaneously.
\end{abstract}

Keyword: Glucose; Concentration; Enzyme; Dielectric loss; Microwave 